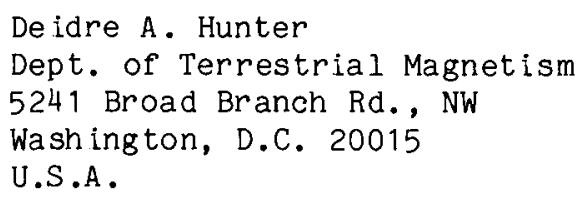

ABSTRACT. A few of the interesting problems concerning star formation in irregular galaxies are reviewed.

\title{
1. The Massive Stars
}

The most obvious stellar component in normal, noninteracting Magellanic-type irregular galaxies are the massive stars. These stars are particularly noticeable in ultraviolet images, such as the dramatic image of the LMC taken during the Apollo 16 mission (Page and Carruthers 1981). The massive stars also produce HII regions which often add to the chaotic optical appearance of the galaxies (cf. de Vaucouleurs and Freeman 1972, Hunter 1982a). The irregulars are, in general, the bluest of the normal disk galaxies, with U-B -0.3 (de Vaucouleurs and de Vaucouleurs 1972; Huchra 1977; de Vaucouleurs, de Vaucouleurs, and Buta 1981), and the blue U-B colors are again indicative of a young massive star population. Unlike the spirals, irregulars are generally bluer in their central regions which is taken to imply a central concentration of the young population.

A small number of irregular galaxies are close enough to be resolved into individual stars. In these galaxies blue and red supergiants have been identified (cf. Humphreys 1980; Hoessel and Danielson 1984; Sandage and Carlson 1985 and references therein), as well as Wolf-Rayet stars (cf. Armandroff and Massey 1985, Massey 1985). The latter, which are evolved from very massive stars, have also been detected from emission features in the integrated spectra of a few more distant irregulars. The numbers or number densities of Wolf-Rayet stars and the spectral-type distributions vary from galaxy to galaxy for reasons which are not yet clear (see Massey 1985), but what is interesting is that evolved massive stars are found in irregulars covering a wide range in luminosity, stellar surface brightness, and hence total star formation rates. There is also some evidence that the massive star luminosity function is similar among different types of galaxies, including irregulars (cf. Freedman 1984; Scalo, preprint). 
Stellar clusters and associations are an important clue to how galaxies form stars. As we heard earlier today, there must be differences in the processes which form dense clusters and those that form 100 se associations (Mathieu, these proceedings). Stellar clusters are quite prominent in many irregulars--both low and $\mathrm{high}$ surface brightness galaxies. In the LMC, for example, clusters covering a full range of ages are found ( $c f$. Hodge 1973, 1980), including old globular-like clusters (cf. Stryker 1983) and the so-called "blue globular clusters" (cf. Hodge 1961). The latter are young dense clusters of $10^{4}-10^{5} \mathrm{M}$. Such clusters are not unique to the LMC. Luminous "superclusters" have been identif ied in other irregulars and spirals (Ables 1971, van den Bergh 1972, Wray and de Vaucouleurs 1980).

However, there is a class of irregulars--the amorphous systems--which are notable for their smooth structure and lack of resolution into stars and clusters (cf. Sandage and Brucato 1979). They do contain Ho-emission, so massive stars are clearly present (cf. Hodge 1966; Hunter 1982a; Gallagher, Hunter, and Knapp 1981), and in some cases kinematically distinct HII regions are found from $\mathrm{high}$ dispersion spectra (Hunter 1982b). Ultraviolet spectra also ind icate the presence of massive stars (Huchra et al. 1983, Lamb et al. 1985). In terms of other global properties, such as surface brightness, luminosity, sizes, kinematics, metallicity, gas-fraction, inferred star-formation rates, and far-IR properties, the amorphous systems are quite similar to the Magellanic-type irregulars (cf. Hunter and Gallagher 1986). But, for unknown reasons the pattern of star formation appears to be different.

\section{Giant Star-Forming Regions}

The irregular galaxies do usually contain a range of sizes of star-forming regions, but they often contain spectaculariy large regions which can be anomalously large for the galaxy's overall distribution in sizes (cf. van den Bergh 1981, Hodge 1983). 30 Doradus in the LMC is the most famous example of a giant star-forming complex. But what is interesting is that the low surface brightness dwarf irregulars can also make giant HII regions. DDO 42, for example, contains an HII complex with the lonizing radiation of hundreds of O stars (cf. Kennicutt, Balick, and Heckman 1980). This raises the interesting question of what sets the limit to the size of a star-forming region in a given galaxian environment. And what are the cloud formation processes that enable a galaxy to put $1 \%$ of its total HI content into a single unit? Elmegreen and Elmegreen (1980) have suggested that stellar bar structures could be important in the formation of glant star-forming regions, and many, although not all, irregulars do have bars.

Giant star-forming regions can also be found in spiral galaxies such as NGC 604 in M33. Compared to giant HII regions in late-type spirals, the large regions in irregulars have similar 
properties--morphology, kinematics, stellar content--in spite of the global differences between spirals and irregulars ( $c f$. Hunter and Gallagher 1985a, Hunter 1982a).

\section{Star Formation as a Cont inuing Process}

Irregular galaxies do not contain only young, massive stars. The smooth underlying background in stellar light (cf. Graham 1982, Hoessel and Danielson 1983), B-V and V-K colors, and JHK colors (Hunter and Gallagher 1985b) indicate the presence of an older ( $\geqq 1$ Gyr) population. In the LMC, stars as old as $10^{10}$ yrs can be ident if ied (cf. Graham 1975, 1977). That is, star formation is a continuing process in these galaxies. Studies of the spatial structure of star formation in irregulars (cf. Hodge 1969, Payne-Gaposhk in 1974, Isserstedt 1984, see also Feitzinger et al. 1981) have shown that the major star formation centers in irregulars move around the galaxy with time in a stochastic manner.

From integrated $\mathrm{H} \alpha$ measurements star-formation rates are deduced which show that irregulars can be quite successful at forming stars, as successful as many spirals (cf. Hunter and Gallagher 1986 and references therein). The $h i g h$ surface brightness irregulars have rates which range from $5 \times 10^{-9}$ to $3 \times 10^{-10}$ with an average of $2 \times 10^{-9}$ $\mathrm{M} / \mathrm{yr} / \mathrm{pc}^{2}$. The spirals observed by Kennicutt (1983) cover the range $6 \times 10^{-8}$ to $5 \times 10^{-10}$ with an average of $5 \times 10^{-9} \mathrm{M} / \mathrm{yr} / \mathrm{pc}^{2}$. Thus, spiral density waves are not necessary to a healthy production of stars.

However, there is a large range in the absolute levels of star formation among the irregulars. Some dwarf irregulars can have rates as low as $10^{-11} \mathrm{M} / \mathrm{yr} / \mathrm{pc}^{2}$. Yet, the global properties, such as color, kinematics, size, metallicity, gas-fraction, star-formation rate per blue luminosity ratio, of all of the irregulars are very similar regardless of the star formation rate (of. Hunter and Gallagher 1986). This raises the question of what properties of a galaxy govern its overall level of star formation activity?

The star formation success of many ir regulars is in spite of differences in the interstellar medium between irregulars and spirals. The irregulars are $r$ ich in gas, but often it is extended far beyond the optical galaxy (cf. van Woerden, Bosma, and Mebold 1975; Huchtmeier, Seiradakis, and Materne 1981). The inner HI gas is lumpy, but the relationship between the current star-forming regions is not always clear (cf. Cottrell 1976; van Gorkom, unpublished). Co luminosities are low relative to spirals (Elmegreen, Elmegreen, and Morris 1980; Israel et al. 1982; Young, Gallagher, and Hunter 1984; Tacconi and Young 1985), indicating something different about the molecular content. As will be discussed further in the next talk (Gallagher, these proceedings), the large dark dust nebulae are far fewer in irregulars and reddenings are lower. The irregulars are also metal poor on the whole relative to spirals (cf. Talent 1980). The 
interesting question here is whether or not these factors affect the star formation processes, and if so, how.

Finally, I would like to say a few words about the star-formation histories of irregulars. The data that are available--time scales to exhaust the gas supplies at the current rate, colors, total stellar masses and luminosities, metallicity, and infrared luminosities--are consistent with constant star formation rates and normal initial mass functions (cf. Lequeux 1979, and discussion and references in Hunter and Gallagher 1986). It is a difficult problem and the uncertainties are large, but so far the data does not force one to invoke bursts of star formation for these non-interacting systems. Statistical fluctuations in the star-formation rate are expected due to the inherent grainy nature of the star-formation process. This will be more severe in dwarf irregulars which support only a few HII regions. But, this is quite different from a coherent global burst of star formation which raises the global star formation level by a large factor over the past rate. In such a burst special processes are impl ied.

Clearly, the irregulars are very interesting and important to our understanding of young stars and star formation processes ( $c f$. Bok 1977 ).

\section{REFERENCES}

Ables, H. D. 1971, Pub. US Nav. Obs., 20, Part 4. Armandroff, T. E. and Massey, P. 1985, Ap. J., 291, 685. Bok, B. 1977, in Structure and Evolution of Normal Galaxies, eds. S. Fall and D. Lynden-Bel l Cambridge Univ. Press), p. 22.

Cottrell, G. A. 1976, M.N.R.A.S., 177, 463.

de Vaucouleurs, G. and de Vaucouleurs, A. 1972, M.N.R.A.S., 77, 1 . de Vaucouleurs, G., de Vaucouleurs, A., and Buta, R. 1981, A. J., 86, 1429.

de Vaucouleurs, G. and Freeman, K. 1972, Vistas Astron., 14, 163. Elmegreen, B., Elmegreen, D., and Morris, M. 1980, Ap. J., 240, 455. Elmegreen, D. M. and Elmegreen, B. 1980, A. J., 85, 1325 .

Feitzinger, J., Glassgold, A., Gerola, H., and Seiden, P. 1981, Astr. Astrophys., 98, 371 .

Freedman, W. 1984, Ph. D. thesis, Univ. of Toronto.

Gallagher, J. S., Hunter, D. A., and Knapp, G. 1981, A. J., 86, 344. Graham, J.A. 1975, P.A.S.P., 87, 641 .

- - 1977, P.A.S.P., 89, 425.

-...... 1982, Ap.J., 252, 474.

Hodge, P. 1961, Ap. J., 133, 413.

…. 1966, Ap. J., 146, 593.

- 1973, A. J.., 78, 807.

..... 1980, $\overline{\mathrm{Ap}} . \mathrm{J} ., 241,125$.

-.... 1983, A. J.. 88, 1323.

Hoessel, J. and Dánielson, G. 1983, Ap. J., 271, 65.

Huchra, J. 1977, Ap. J. Suppl., 35, 171. 
Huchra, J., Geller, M., Gallagher, J., Hunter, D., Harmann, L., et al. 1983, Ap. J., 274, 125.

Huchtme ier, W. K., Seiradarkis, J. H., and Materne, J. 1980, Astr. Astrophys., 91, 341 .

Humphreys, R.M. 1980, Ap. J., 238, 65 .

Hunter, D. A. 1982a, Ap. J., 260, 81 .

- 1982b, Ph.D. thesis, Univ. of Illinois.

Hunter, D. A. and Gallagher, J. S. 1985a, A. J., 90, 80.

-..... 1985b, A. J., 90, 1457.

-.... 1986, P.A.S.P., in press.

Israel, F. P., de Graauw, T., Lidholm, S., van de Stadt, H., and de Vries, C. 1982, Ap. J., 262, 100 .

Kennicutt, R. 1983, Ap. J., 272, 54 .

Kennicutt, R., Balick, B., and Heckman, T. 1980, P.A.S.P., 92, 134.

Lamb, S., Gallagher, J., Hjellming, M., and Hunter, D. 1985, Ap. J., $291,63$.

Lequeux, J. 1979, Astr. Astrophys., 71, 1.

Massey, P. 1985, P.A.S.P., 97, 5 .

Page, T. and Carruthers, G. R. 1981, Ap. J., 248, 906.

Sandage, A. and Brucato, R. 1979, A. $\overline{J_{.}}, \overline{84}, 472$.

Sandage, A. and Carlson, G. 1985, A. J., 90, 1019.

Stryker, L. L. 1983, Ap. J., 266, $\overline{82}$.

Tacconi, L. J. and Young, J. 1985, Ap. J., 290, 602.

Talent, D. 1980 , Ph.D. thesis, Rice University.

van den Bergh, S. 1972, J. R. Astron. Soc. Can., 66, 237.

-...... 1981, A. J., 86, $14 \overline{64}$.

van Woerden, H., Bosma, A., and Mebold, U. 1975, in La Dynamique des Galaxies Spirales, ed. L. Weliachew (Paris:CNRS), p. 483 .

Wray, J. D. and de Vaucouleurs, G. 1980, A. .J, 85, 1 .

Young, J., Gallagher, J., and Hunter, D. 1984, Ap. J., 276, 476. 